\title{
Predictors of Cardioembolic Stroke in Japanese Patients with Atrial Fibrillation in the Fushimi AF Registry
}

\author{
Ken Yasuda ${ }^{a}$ b Shunichi Fukuda ${ }^{c}$ Michikazu Nakamura ${ }^{a}$ Ryo Ohtani ${ }^{a}$ \\ Yasuhiro Kuwata $^{a}$ Masaki Takata ${ }^{a}$ Makoto Sainouchi ${ }^{a}$ Masahiro Gotou ${ }^{a}$

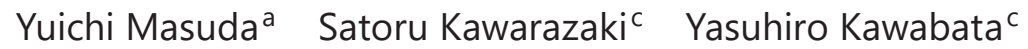 \\ Nagako Murase $^{a}$ Tomokazu Aokic Naohiro Yonemoto $^{\mathrm{e}}$ \\ Masaharu Akao ${ }^{d}$ Tetsuya Tsukaharac \\ aDepartment of Neurology, National Hospital Organization Kyoto Medical Center, Kyoto, \\ Japan; ${ }^{b}$ Department of Neurology, Kyoto University, Graduate School of Medicine, Kyoto, \\ Japan; ' Department of Neurosurgery, National Hospital Organization Kyoto Medical Center, \\ Kyoto, Japan; ${ }^{d}$ Department of Cardiology, National Hospital Organization Kyoto Medical \\ Center, Kyoto, Japan; e Department of Biostatistics, School of Public Health, Kyoto University, \\ Kyoto, Japan
}

\section{Keywords}

Atrial fibrillation · Cardioembolic stroke $\cdot$ Risk factors · Stroke subtype $\cdot$ Infarct volume

Cohort study

\section{Abstract}

Background: Large-scale clinical trials have analyzed risk factors for any ischemic stroke in patients with atrial fibrillation (AF). However, the risk factors for cardioembolic stroke (CES), specifically, have not been reported. To clarify the risk factors for CES and clinically significant cardioembolic infarction, we examined the incidence of CES and larger infarct volume (IV) (>30 mL) CES, employing the Fushimi AF Registry, a community-based prospective cohort of AF patients in the Fushimi ward, Kyoto, Japan. Methods: A total of 4,182 Fushimi AF patients were enrolled from March 2011 to December 2014. The risk factors for CES were evaluated using multivariate analysis. Results: Of 4,182 patients enrolled, 3,749 patients were observed for $\geq 1$ year. During the follow-up period (mean duration, $979 \pm 7.7$ days), 91/3,749 patients experienced a CES (2.43\%). Significant risk factors associated with CES were older age (odds ratio $[\mathrm{OR}], 1.31 ; 95 \%$ confidence interval $[\mathrm{Cl}], 1.01-1.72 ; p=0.046)$, low body weight (OR, 1.30; $95 \% \mathrm{Cl}, 1.03-1.65 ; p=0.033)$, sustained $\mathrm{AF}(\mathrm{OR}, 1.67 ; 95 \% \mathrm{Cl}, 1.05-2.71 ; p=0.034)$, and previ- 
ous stroke or transient ischemic attack (TIA) $(\mathrm{OR}, 1.94 ; 95 \% \mathrm{Cl}, 1.22-3.06 ; p=0.004)$. Predictors of a large IV were chronic kidney disease (CKD) $(\mathrm{OR}, 2.08 ; 95 \% \mathrm{Cl}, 1.09-4.05 ; p=0.027)$ and previous stroke/TIA (OR, 2.27; $95 \% \mathrm{Cl}, 1.19-4.24 ; p=0.011)$. Conclusions: In this populationbased cohort of Japanese patients with $A F$, in addition to previous stroke/TIA and older age, sustained AF and low body weight emerged as risk factors for CES, as opposed to any stroke, which may have a different risk profile. Patients with CKD or previous stroke/TIA who developed cardioembolic infarction exhibited more advanced severity. There is a need for direct oral anticoagulants that can be used safely in patients with comorbid AF and CKD.

(C) 2018 The Author(s)

Published by S. Karger AG, Basel

\section{Introduction}

Atrial fibrillation (AF), which is the most common type of arrhythmia, increases the risk of thromboembolic events [1], and in particular, cardioembolic stroke (CES), which is the severest ischemic stroke subtype. Stroke risk has been examined in patients with AF in several studies [2-6], wherein various risk score rubrics were advocated, such as the $\mathrm{CHADS}_{2}$ scoring system [3] and, more recently, the $\mathrm{CHA}_{2} \mathrm{DS}_{2}$-VASc scoring system [4]. However, these scoring systems assess the risk for any stroke, including noncardioembolic ischemic stroke (i.e., lacunar or atherothrombotic infarction) and/or hemorrhagic stroke (i.e., cerebral, subarachnoid, or chronic subdural hemorrhage), without specificity for CES [5, 6]. This may be partly because diagnostic imaging examinations were not usually performed at the onset of stroke in these clinical studies. Thus, it is unclear what factors are closely associated with CES in AF patients. For the same reason, the risk factors associated with infarct volume (IV) have not been determined in most clinical studies either, although the clinical outcome, as indexed by clinical stroke scales, has been found to be correlated with the lesion volume [7].

The Fushimi ward is located in southern Kyoto, Japan. It is a densely populated region with a total population of 283,000 and can be considered representative of a typical urban community in Japan. The Fushimi AF Registry includes a community-based prospective cohort of Japanese patients with AF. Investigators have already developed several lines of evidence based on this resource [8-11].

The aims of this study were (1) to identify predictors specific to CES in Japanese AF patients and (2) to clarify risk factors associated with clinically meaningful large cardioembolic IV. Towards these aims, we used the Fushimi AF Registry to enroll all AF patients in the Fushimi ward in our analysis of stroke incidence and risk factors [8].

\section{Materials and Methods}

For further details, refer to online supplementary methods (for all online suppl. material, see www.karger.com/doi/10.1159/000488206).

\section{Fushimi AF Registry Data Sources}

The detailed study design, patient enrollment, definition of the measurements, and Fushimi AF Registry subjects' baseline clinical characteristics have been described previously. All Fushimi AF Registry patients who had undergone 12-lead electrocardiography or Holter monitoring at any of the 80 participating institutions (all Fushimi Ishi-Kai [Fushimi Medical Association] members) since March 2011 were consecutively enrolled without exclusion. Patients without at least 1 year of follow-up were excluded. Most of the patients had undergone brain magnetic resonance imaging (MRI) examinations at stroke onset, 


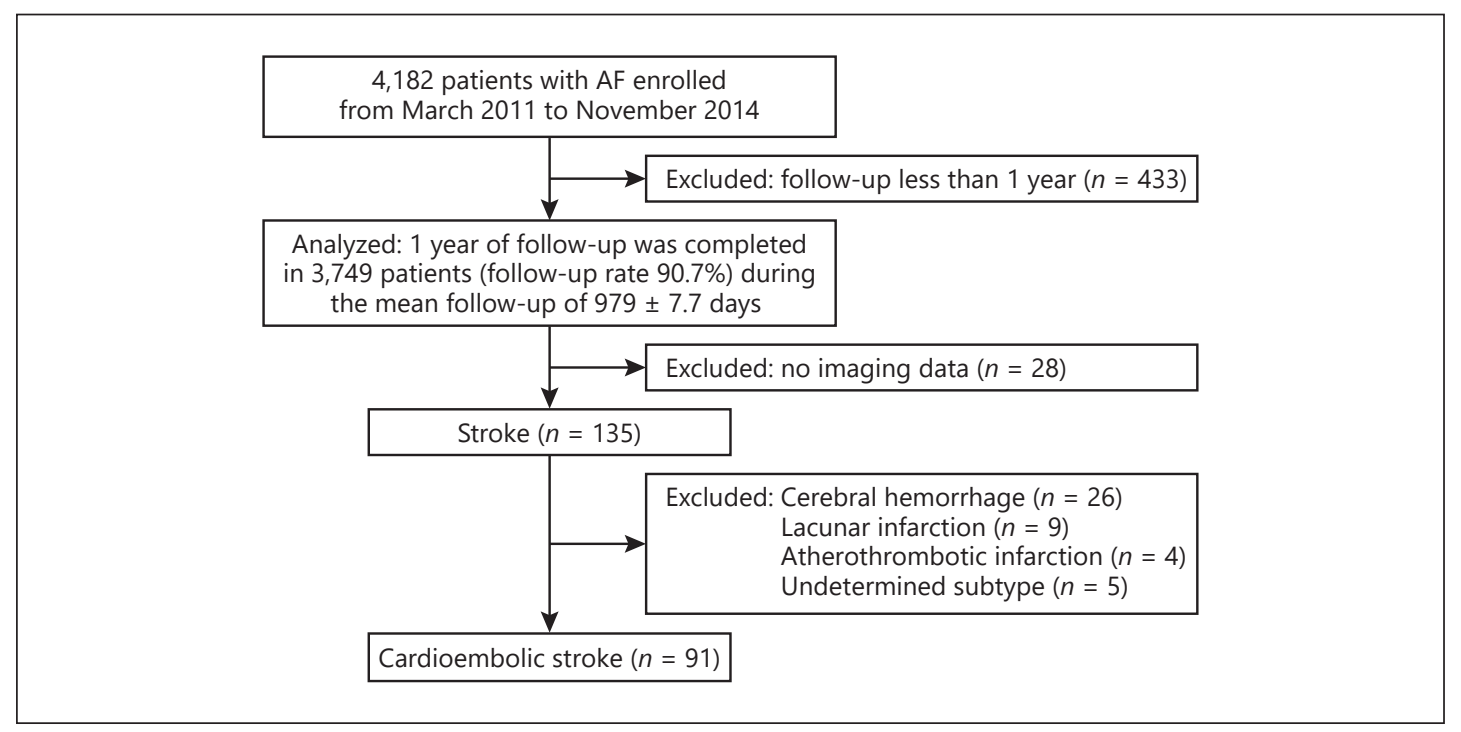

Fig. 1. Flowchart of this study.

enabling us to determine the ischemic stroke type and measure IV with diffusion-weighted imaging. Cases for which MRI or computed tomography (CT) data from stroke events were unavailable were excluded (Fig. 1).

The study protocol conformed to the ethical guidelines of the 2000 Declaration of Helsinki and was approved by the ethics committees of both the National Hospital Organization Kyoto Medical Center and Ijinkai Takeda General Hospital.

\section{Stroke Ascertainment and Definitions}

We identified stroke cases and determined stroke subtypes based on brain MRI or CT images obtained at stroke onset. Because all registry patients had cardiac-originated AF, we could not use a standard classification. Several independent neurologists reviewed each case and confirmed the stroke diagnoses, as informed by the literature [12]. The underlying stroke etiology was classified as CES, large-artery atherosclerosis, lacunar infarction, or undetermined etiology (see suppl. methods). Chronic kidney disease (CKD) was defined as estimated glomerular filtration rate $<60 \mathrm{~mL} / \mathrm{min} / 1.73 \mathrm{~m}^{2}$.

\section{Measurement}

IVs were measured manually in diffusion-weighted imaging or CT images, section by section, using the ITK-SNAP 2.2.0 software (http://www.itksnap.org/pmwiki/pmwiki. php?n=Main.Downloads). IV was classified as large $(>30 \mathrm{~mL})$ or small $(\leq 30 \mathrm{~mL})$, based on a study that showed that lesion volume is correlated with functional status [13]. Clinical background data were compared between the resulting large and small IV groups.

\section{Statistical Analysis}

Means are reported with standard deviations. We compared binary and categorical variables using the $\chi^{2}$ test when appropriate; otherwise, the Fisher exact test was applied. We compared continuous variables using the Student $t$ test or the Wilcoxon rank-sum test. We performed univariate and multivariate logistic regression for identified risk factors and calculated odds ratios (ORs) and 95\% confidence intervals (CIs). A set of potential risk factors was chosen a priori based on biological plausibility and a priori knowledge [2-6]. A two-sided 
Table 1. Comparison of baseline clinical characteristics between patients with and without CES

\begin{tabular}{|c|c|c|c|c|c|}
\hline & CES & CES large IV & CES small IV & Non-CES & $p$ value \\
\hline Number & 91 & 45 & 46 & 3,658 & \\
\hline Male & $45(49.5)$ & $22(24.2)$ & $23(25.3)$ & $2,178(59.5)$ & 0.053 \\
\hline Age, years & $78.9 \pm 8.4$ & $79.6 \pm 1.63$ & $78.1 \pm 1.61$ & $73.5 \pm 11.0$ & $<0.001$ \\
\hline Height, $\mathrm{cm}$ & $156.1 \pm 10.8$ & $155.3 \pm 1.5$ & $156.9 \pm 1.5$ & $160.0 \pm 10.1$ & $<0.001$ \\
\hline Body weight, kg & $53.5 \pm 12.7$ & $52.3 \pm 2.0$ & $54.8 \pm 2.0$ & $59.3 \pm 13.2$ & $<0.001$ \\
\hline BMI & $21.7 \pm 3.7$ & $21.5 \pm 0.6$ & $22.1 \pm 0.6$ & $23.1 \pm 4.0$ & 0.003 \\
\hline Systolic blood pressure, mm Hg & $121.4 \pm 21.5$ & $118.0 \pm 2.8$ & $124.7 \pm 2.8$ & $124.8 \pm 19.0$ & 0.092 \\
\hline Diastolic blood pressure, $\mathrm{mm} \mathrm{Hg}$ & $67.5 \pm 15.3$ & $64.0 \pm 1.9$ & $70.9 \pm 1.9$ & $70.7 \pm 12.8$ & 0.018 \\
\hline Sustained AF & $61(67.3)$ & $31(34.0)$ & $30(33.3)$ & $1,851(50.6)$ & 0.002 \\
\hline Paroxysmal AF & $30(33.0)$ & $14(15.3)$ & $16(17.7)$ & $1,807(49.4)$ & 0.002 \\
\hline \multicolumn{6}{|l|}{ Comorbidities } \\
\hline $\mathrm{CHADS}_{2}$ score & $2.69 \pm 1.30$ & $2.76 \pm 0.20$ & $2.63 \pm 0.20$ & $2.01 \pm 1.33$ & $<0.001$ \\
\hline $\mathrm{CHA}_{2} \mathrm{DS}_{2}$-VASc score & $4.28 \pm 1.60$ & $4.31 \pm 0.25$ & $4.26 \pm 0.25$ & $3.34 \pm 1.70$ & $<0.001$ \\
\hline Congestive heart failure & $35(38.5)$ & $18(19.7)$ & $17(18.8)$ & $973(26.6)$ & 0.012 \\
\hline Hypertension & $59(64.8)$ & $30(33.0)$ & 29 (31.8) & $2,270(62.1)$ & 0.589 \\
\hline Diabetes mellitus & $19(21.0)$ & $5(5.5)$ & $14(15.5)$ & $841(23.0)$ & 0.636 \\
\hline Dyslipidemia & $35(38.5)$ & $16(17.6)$ & 19 (20.9) & $1,583(43.3)$ & 0.360 \\
\hline Chronic kidney disease & $46(50.6)$ & $26(28.6)$ & $20(22.0)$ & 1,277 (34.9) & 0.002 \\
\hline eGFR & $46.2 \pm 17.5$ & $44.9 \pm 3.1$ & $47.3 \pm 3.0$ & $51.2 \pm 20.7$ & 0.022 \\
\hline Hemoglobin, g/dL & $12.4 \pm 2.0$ & $12.0 \pm 0.30$ & $12.8 \pm 0.30$ & $12.9 \pm 2.02$ & 0.014 \\
\hline Previous stroke/TIA & $33(36.3)$ & $18(19.7)$ & $15(16.6)$ & 707 (19.3) & $<0.001$ \\
\hline Dilated cardiomyopathy & $0(0)$ & $0(0)$ & $0(0)$ & $51(1.39)$ & \\
\hline Hypertrophic cardiomyopathy & $3(3.3)$ & $1(1.1)$ & $2(2.2)$ & $37(1.01)$ & 0.036 \\
\hline Ejection fraction & $63.0 \pm 10.7$ & $63.5 \pm 1.9$ & $62.4 \pm 1.8$ & $63.0 \pm 11.7$ & 0.603 \\
\hline Left atrial dilation, $\mathrm{mm}$ & $46.4 \pm 7.6$ & $46.8 \pm 1.4$ & $46.1 \pm 1.32$ & $43.7 \pm 8.3$ & 0.006 \\
\hline OAC prescription at baseline & $54(59.3)$ & $29(31.9)$ & $25(27.4)$ & $1,944(53.4)$ & 0.262 \\
\hline Warfarin & $50(55.0)$ & $26(28.5)$ & $24(26.5)$ & $1,678(46.1)$ & 0.095 \\
\hline Dabigatran & $3(3.3)$ & $2(2.2)$ & $1(1.1)$ & $133(3.64)$ & 0.864 \\
\hline Rivaroxaban & $1(1.1)$ & $1(1.1)$ & $0(0)$ & $84(2.3)$ & 0.449 \\
\hline Apixaban & $0(0)$ & $0(0)$ & $0(0)$ & $49(1.34)$ & \\
\hline $\begin{array}{l}\text { Antiplatelet drugs prescription at } \\
\text { baseline }\end{array}$ & $41(45.1)$ & $20(22.0)$ & $21(23.1)$ & $1,041(27.7)$ & $<0.001$ \\
\hline
\end{tabular}

Categorical data are presented as number (\%) and continuous data as mean \pm SD. We compared categorical variables using the $\chi^{2}$ test when appropriate; otherwise, we used the Fisher exact test. Continuous variables were compared using the Student $t$ test or Wilcoxon rank-sum test based on the distribution. $p$ values were used for comparison between CES and non-CES. CKD was defined as eGFR $<60 \mathrm{~mL} / \mathrm{min} / 1.73 \mathrm{~m}^{2}$. Sustained AF referred to persistent and permanent AF. Infarct volume was classified as large $(>30 \mathrm{~mL})$ or small $(\leq 30 \mathrm{~mL})$. AF, atrial fibrillation; BMI, body mass index; CES, cardioembolic stroke; CI, confidence interval; CKD, chronic kidney disease; eGFR, estimated glomerular filtration rate; IV, infarct volume; OAC; oral anticoagulant; TIA, transient ischemic attack.

$p$ value $<0.05$ was considered statistically significant. The rationales for risk factor selection are described in the online supplementary methods. All analyses were performed with JMP, version 11 (SAS Institute, Cary, NC, USA).

\section{Results}

Of 4,182 patients that were enrolled from March 2011 to December 2014, 3,749 patients were observed for at least 1 year (follow-up rate, 90.7\%). The mean follow-up period for these 3,749 patients was $979 \pm 7.7$ days (median, 1,099 days; range 553-1,470 days). CES occurred in 91/3,749 (2.43\%) during follow-up. Twenty-eight cases for which we could not obtain MRI or CT data were excluded. 
Table 2. Predictors of CES during follow-up: multivariate analysis after adjustment for $\mathrm{CHADS}_{2}$ score components, body weight loss (per $10 \mathrm{~kg}$ ), prevalence of sustained AF, CKD, and OAC prescription at baseline

\begin{tabular}{llll}
\hline Variable $(n=3,343)$ & Odds ratio & $95 \% \mathrm{CI}$ & $p$ value \\
\hline Age (per 10 years) & 1.31 & $1.01-1.72$ & 0.046 \\
Body weight loss (per 10 kg) & 1.30 & $1.03-1.65$ & 0.033 \\
Previous stroke/TIA & 1.94 & $1.22-3.06$ & 0.004 \\
Hypertension & 1.00 & $0.64-1.60$ & 0.969 \\
Congestive heart failure & 1.06 & $0.66-1.70$ & 0.797 \\
Diabetes mellitus & 0.92 & $0.53-1.53$ & 0.754 \\
Sustained AF & 1.67 & $1.05-2.71$ & 0.034 \\
OAC prescription at baseline & 1.10 & $0.70-1.74$ & 0.676 \\
Chronic kidney disease & 1.52 & $0.96-2.41$ & 0.072 \\
Male & 1.04 & $0.63-1.76$ & 0.857 \\
\hline
\end{tabular}

$\mathrm{AF}$, atrial fibrillation; CES, cardioembolic stroke; CI, confidence interval; CKD, chronic kidney disease; OAC, oral anticoagulant; TIA, transient ischemic attack.

Table 3. Predictors of large infarct volume CES ( $\geq 30 \mathrm{~mL}$ ) during follow-up: multivariate analysis after adjustment for $\mathrm{CHADS}_{2}$ score components, sex, body weight loss (per $10 \mathrm{~kg}$ ), prevalence of sustained AF, CKD, and $\mathrm{OAC}$ prescription at baseline

\begin{tabular}{|c|c|c|c|c|c|c|}
\hline \multirow[t]{2}{*}{ Variable $(N=3,343)$} & \multicolumn{3}{|c|}{ CES large IV } & \multicolumn{3}{|c|}{ CES small IV } \\
\hline & $\begin{array}{l}\text { odds } \\
\text { ratio }\end{array}$ & $95 \% \mathrm{CI}$ & $p$ value & $\begin{array}{l}\text { odds } \\
\text { ratio }\end{array}$ & $95 \% \mathrm{CI}$ & $p$ value \\
\hline Age (per 10 years) & 1.34 & $0.92-1.99$ & 0.141 & 1.29 & $0.91-1.88$ & 0.166 \\
\hline Body weight loss (per $10 \mathrm{~kg}$ ) & 1.40 & $1.00-2.00$ & 0.054 & 1.21 & $0.88-1.68$ & 0.256 \\
\hline Previous stroke/TIA & 2.27 & $1.19-4.24$ & 0.011 & 1.65 & $0.83-3.12$ & 0.138 \\
\hline Hypertension & 1.15 & $0.61-2.26$ & 0.662 & 0.88 & $0.47-1.69$ & 0.700 \\
\hline Congestive heart failure & 0.96 & $0.49-1.84$ & 0.894 & 1.18 & $0.60-2.27$ & 0.620 \\
\hline Diabetes mellitus & 0.42 & $0.14-0.99$ & 0.074 & 1.60 & $0.80-3.05$ & 0.167 \\
\hline Sustained AF & 1.86 & $0.96-3.81$ & 0.074 & 1.50 & $0.79-2.93$ & 0.220 \\
\hline OAC prescription at baseline & 1.33 & $0.70-2.59$ & 0.393 & 0.92 & $0.49-1.74$ & 0.798 \\
\hline Chronic kidney disease & 2.08 & $1.09-4.05$ & 0.027 & 1.12 & $0.58-2.13$ & 0.737 \\
\hline Male & 1.16 & $0.56-2.42$ & 0.691 & 0.96 & $0.47-1.96$ & 0.903 \\
\hline
\end{tabular}

AF, atrial fibrillation; CES, cardioembolic stroke; CI, confidence interval; CKD, chronic kidney disease; IV, infarct volume; OAC, oral anticoagulant; TIA, transient ischemic attack.

\section{Baseline Clinical Characteristics}

The baseline clinical characteristics of the 91 patients who experienced CES are summarized and are compared with the characteristics of non-CES patients (Table 1). Compared to those without CES, AF patients with CES tended to be older, shorter, and with a lower body weight, and were more likely to have a history of heart failure, prior stroke/transient ischemic attack (TIA), CKD, hypertrophic cardiomyopathy, and sustained AF. The average $\mathrm{CHADS}_{2}$ and $\mathrm{CHA}_{2} \mathrm{DS}_{2}$-VASc scores were higher while average estimated glomerular filtration rate and hemoglobin levels were lower in CES than in non-CES patients. CES patients were less likely to be taking an oral anticoagulant (OAC) than non-CES patients, with the most common OAC being warfarin (alternative OACs were limited when the majority of patients were enrolled). 


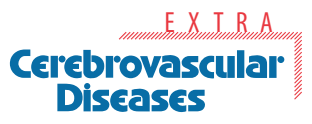

Table 4. CES case characteristics

\begin{tabular}{l|l}
\hline Cerebrovasc Dis Extra 2018;8:50-59 \\
\hline DOI: 10.1159/000488206 & $\begin{array}{l}\text { @ } 2018 \text { The Author(s). Published by S. Karger AG, Basel } \\
\text { www.karger.com/cee }\end{array}$ \\
\hline
\end{tabular}

Yasuda et al.: Predictors of Cardioembolic Stroke in AF Patients

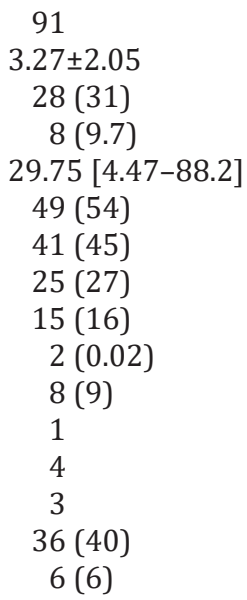
Categorical data are presented as number (\%) and continuous data
as mean \pm SD. ICA/M1 occlusion were disclosed on magnetic resonance
imaging at the onset. CES, cardioembolic stroke; CKD, chronic kidney
disease; DOAC, direct oral anticoagulant; ICA, internal carotid artery;
M1, M1 segment of middle cerebral artery; mRS, modified Rankin Scale;
OAC, oral anticoagulant; PT-INR, prothrombin time-international nor-
malized ratio; rt-PA, recombinant tissue plasminogen activator.

Compared to patients with a smaller IV ( $\leq 30 \mathrm{~mL}$ ), patients with a larger IV ( $>30 \mathrm{~mL})$ were, on average, older, of lower body weight, and more likely to have a history of heart failure, previous stroke/TIA, or CKD.

\section{Univariate and Multivariate Analysis}

The univariate analysis results for CES and IV are reported in online supplementary Tables 1 and 2. Subsequent multivariate analysis after adjustment indicated that age (per decade; OR, 1.31; 95\% CI, 1.10-1.72), low body weight (LBW; i.e., body mass $\leq 50 \mathrm{~kg}$ ) (OR, 1.30; 95\% CI, 1.03-1.65), sustained AF (OR, 1.67; 95\% CI, 1.05-2.71), and previous stroke/ TIA (OR, 1.94; 95\% CI, 1.22-3.06) were risk factors for CES (Table 2). CKD (OR, 2.08; 95\% CI, 1.09-4.05) and previous stroke/TIA (OR, 2.27; 95\% CI, 1.19-4.24) were found to be associated with a larger IV (Table 3).

\section{CES Cases}

Of the 91 patients who experienced CES during the follow-up period (Table 4), 8 (9.7\%) received recombinant tissue plasminogen activator (rt-PA); the mean modified Rankin Scale score of these patients was $3.27 \pm 2.05$. Notably, 41 of the 91 CES patients $(45 \%)$ received warfarin at the time of stroke onset, although only 34 (37\%) had a follow-up prothrombin time-international normalized ratio (PT-INR) value maintained within the warfarin therapeutic target range as established in the Japanese guidelines (PT-INR of 1.6-2.6 for patients $\geq 70$ years old and PT-INR of 2.0-3.0 for patients $<70$ years old) [14]. Most of the remaining patients $(61 \%)$ had a PT-INR under the therapeutic range, indicating OAC underdosage. Sixteen (39\%) of the patients were not administered an OAC at the time of CES onset. 

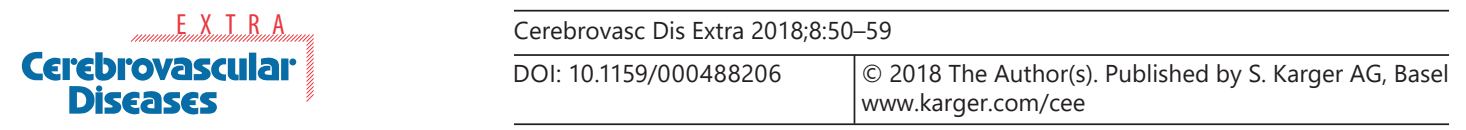

Yasuda et al.: Predictors of Cardioembolic Stroke in AF Patients

\section{Discussion}

Predictors of CES

Predictors of CES identified in this study were of an older age, had an LBW, sustained AF, and prior stroke/TIA.

\section{Sustained AF}

Several studies have reported that patients with paroxysmal AF have a risk of thromboembolic events similar to those with sustained (persistent and permanent) AF [6]. However, a recent meta-analysis reported that patients with sustained AF had a higher rate of stroke/ systemic embolism than patients with paroxysmal AF [15]. AF events lasting longer than 1 day have been shown to be independently associated with embolism, and AF burden (i.e., time spent in AF) has been shown to be associated with an increased risk of stroke [16]. AF presence, duration, and burden status data may be integrated to obtain improved clinical risk stratification with continuous arrhythmia burden monitoring [17]. A previous study based on a Fushimi AF Registry cohort showed that sustained AF was independently associated with a higher incidence of stroke/systemic embolism than paroxysmal AF [9]. The present findings suggest that patients with sustained AF are also at a greater risk than patients with paroxysmal AF for CES, in particular.

\section{Low Body Weight}

In the Fushimi AF Registry, patients with LBW (body mass $\leq 50 \mathrm{~kg}$ ) had high-risk profiles and showed a higher incidence of any stroke and systemic embolism [10]. The inverse relationship between obesity and prognosis in AF patients has been termed an "obesity paradox." Several large studies have found that AF patients who were overweight (body mass index, 25-30) or obese (body mass index, $\geq 30$ ) tended to have a better prognosis in terms of cardiovascular hospitalizations, global mortality risk, and cardiovascular mortality risk [18]. We also found that LBW was a risk factor for CES in Japanese AF patients. Hence, there may be an inverse relationship between body weight and prognosis in AF patients, which extends beyond the normal weight versus obesity range into the LBW versus normal body weight range of comparison.

Asian AF patients are generally leaner and smaller than European and North American AF patients; relative to Europeans and North Americans in the RE-LY [19], ROCKET-AF [20], and ARISTOTLE [21] studies, Asian patients were, on average, 20,16 and $17 \mathrm{~kg}$ lighter, respectively. The proportions of Fushimi AF Registry enlistees with a body weight $<50$ and $<60 \mathrm{~kg}$ have been reported to be 25.7 and $55.0 \%$, respectively [8]. According to the National Health and Nutrition Survey, the mean body weights of Japanese men and women over 70 years old in 2014 were 50.7 and $50.1 \mathrm{~kg}$, respectively (http://www.mhlw.go.jp/).

There are several possible explanations for the association of LBW with a higher risk of stroke [22]. First, LBW may be due to poor nutritional status or illness-related weight loss (e.g., malignancy, chronic obstructive pulmonary disease, and gastrointestinal disease). Second, underweight patients may have advanced atrial fibrosis and remodeling involving the activation of the renin-angiotensin system.

\section{Other Risk Factors}

Advanced age and previous stroke/TIA are widely recognized as risk factors for stroke in patients with AF, including in the $\mathrm{CHADS}_{2}$ rubric [3] and $\mathrm{CHADS}_{2}$-VASc rubric [4]. The present study confirmed these two factors as independent risk factors for CES. In contrast, diabetes mellitus, hypertension, or congestive heart failure, which are commonly listed as risk factors for any ischemic stroke in AF patients, were not found to be significant risk factors for CES. 
In a previous study, diabetes mellitus was associated with a significantly increased risk of AF (+26\%) among women, but not among men [23]. It is still controversial whether diabetes mellitus is [2] or is not [23] a risk factor even for any ischemic stroke in patients with AF. The present study supports the view that diabetes mellitus is not a risk factor for CES.

Hypertension was not identified as an independent predictor of CES in the present study despite its common description as an important risk factor for any stroke. It may be that hypertension is associated with atherothrombotic cerebral infarction, lacunar infarction, hypertensive cerebral hemorrhage, and subarachnoid hemorrhage, but not with CES. Another possibility is that blood pressure (BP) was well controlled in the Fushimi AF Registry cohort (systolic BP $124.7 \pm 19.1 \mathrm{~mm} \mathrm{Hg}$, diastolic BP $70.6 \pm 12.8 \mathrm{~mm} \mathrm{Hg}, n=3,719$ ). Future investigations are needed to clarify the possible role of hypertension in CES.

\section{Predictors of IV}

IV has been proposed as an alternative criterion (surrogate endpoint) to classical disability scales [24]. However, IV has not been associated with predictors of disability. The present study, to our knowledge, is the first to demonstrate that CKD and previous stroke/ TIA are significant predictors of a larger IV. Notably, LBW, sustained AF, hypertension, and diabetes mellitus were not identified as significant risk factors for a larger IV.

CKD is characterized by endothelial dysfunction and increased coagulation in association with factor VIII activity [25]. The abnormal hemostatic profiles of patients with CKD may be related to an elevated risk of thrombotic events. Thus, CKD has been associated with an increased risk of stroke or systemic thromboembolism among patients with AF [5]. The mechanism of this increased risk may be related to anemia, oxidative stress, elevated plasma asymmetrical dimethylarginine (an inhibitor of nitric oxide synthesis), inflammation, and coagulation-promoting conditions [26].

Therefore, anticoagulation therapy should be sufficiently performed for AF patients with CKD. However, there are concerns regarding OAC prescriptions in these patients because of the increased risk of bleeding associated with CKD [27], which may lead to OAC underuse or underdosage. In addition, the metabolism of direct OAC drugs is largely dependent on the kidneys for elimination and little is known regarding the safety and efficacy of these drugs in patients with a creatinine clearance rate $<25 \mathrm{~mL} / \mathrm{min}$ because they were excluded from all pivotal phase-3 direct OAC trials [27]. Because direct OAC use is often restricted in patients with advanced CKD, it has not been shown which direct OACs, if any, are safe for CKD patients at risk of large infarction [27].

\section{CES Cases}

Kim et al. [12] found that $81.2 \%$ of ischemic stroke patients had AF-related stroke. Similarly, in the present study, CES patients accounted for $67.4 \%$ of all stroke patients and $83.5 \%$ of all ischemic stroke patients. The proportion of patients receiving rt-PA was larger in the present study than in other studies $[28,29]$, perhaps because Japan has a relatively high MRI delivery capacity (http://www.oecd.org/), with a large portion of hospitals capable of supporting rt-PA therapy, particularly in the Fushimi ward, where these capacities exceed the national average. Accordingly, it might be that education for stroke in the Fushimi ward has been widely dispersed among our patients.

In the present study, the absence of an OAC prescription was not a significant risk factor. This negative finding may be related to inappropriate OAC use in the study region. The Fushimi AF Registry has been demonstrated to encompass inappropriate OAC use in AF management, including OAC overuse among low-risk patients, OAC underuse among at-risk patients, and underdosing of warfarin [30]. Such inappropriate OAC use may be related to the narrow therapeutic range of OACs, drug and food interactions, the need for better monitoring, 
and/or concerns regarding bleeding risk [8]. Regardless, there is discordance between recommended guidelines and real-world clinical practice.

\section{Limitations}

This study has several limitations. First, there were a limited number of stroke patients in the study population during the observation period. Second, antithrombotic drugs and doses were determined at the discretion of the attending physician. These limitations are intrinsic to most large multicenter registry studies.

\section{Conclusions}

In this population-based cohort of Japanese patients with $\mathrm{AF}$, older age, sustained $\mathrm{AF}$, previous stroke/TIA, and LBW were independently associated with the incidence of CES, as opposed to any stroke, which may have a different risk profile. Patients with CKD or previous stroke/TIA who developed cardioembolic infarction exhibited more advanced severity. There is a need for direct OACs that can be used safely in patients with comorbid AF and CKD.

\section{Acknowledgements}

We sincerely appreciate the help of all of the institutions that participated in the Fushimi AF Registry and the contributions of the institutions' clinical research coordinators (T. Shinagawa, M. Mitamura, M. Fukahori, M. Kimura, M. Fukuyama, and C. Kamata).

\section{Disclosure Statement}

The Fushimi AF Registry is supported by research funding from Boehringer Ingelheim, Bayer Healthcare, Pfizer, Bristol-Myers Squibb, Astellas Pharma, AstraZeneca, Daiichi-Sankyo, Novartis Pharma, MSD, Sanofi-Aventis, and Takeda Pharmaceutical. This research was supported in part by the Practical Research Project for Lifestyle-Related Diseases, including cardiovascular diseases and diabetes mellitus, from the Japanese Agency for Medical Research and Development, AMED (18ek0210082h0002, 18ek0210056h0003). Dr. Akao has received lecture fees from Pfizer, Bristol-Myers Squibb, Boehringer Ingelheim, Bayer Healthcare, and Daiichi-Sankyo.

\section{References}

1 Wolf PA, Abbott RD, Kannel WB: Atrial fibrillation as an independent risk factor for stroke: the Framingham study. Stroke 1991;22:983-988.

2 Stroke Risk in Atrial Fibrillation Working Group: Independent predictors of stroke in patients with atrial fibrillation: a systematic review. Neurology 2007;69:546-554.

3 Gage BF, Waterman AD, Shannon W, Boechler M, Rich MW, Radford MJ: Validation of clinical classification schemes for predicting stroke: results from the national registry of atrial fibrillation. JAMA 2001;285:28642870.

4 Lip GY, Frison L, Halperin JL, Lane DA: Identifying patients at high risk for stroke despite anticoagulation: a comparison of contemporary stroke risk stratification schemes in an anticoagulated atrial fibrillation cohort. Stroke 2010;41:2731-2738.

5 Olesen JB, Lip GY, Kamper AL, Hommel K, Kober L, Lane DA, et al: Stroke and bleeding in atrial fibrillation with chronic kidney disease. N Engl J Med 2012;367:625-635. 
6 Hohnloser SH, Pajitnev D, Pogue J, Healey JS, Pfeffer MA, Yusuf S, et al: Incidence of stroke in paroxysmal versus sustained atrial fibrillation in patients taking oral anticoagulation or combined antiplatelet therapy: an ACTIVE W substudy. J Am Coll Cardiol 2007;50:2156-2161.

7 Tong DC, Yenari MA, Albers GW, O’Brien M, Marks MP, Moseley ME: Correlation of perfusion- and diffusionweighted MRI with NIHSS score in acute $(<6.5 \mathrm{~h})$ ischemic stroke. Neurology 1998;50:864-870.

8 Akao M, Chun YH, Wada H, Esato M, Hashimoto T, Abe M, et al: Current status of clinical background of patients with atrial fibrillation in a community-based survey: the Fushimi AF registry. J Cardiol 2013;61:260-266.

9 Takabayashi K, Hamatani Y, Yamashita Y, Takagi D, Unoki T, Ishii M, et al: Incidence of stroke or systemic embolism in paroxysmal versus sustained atrial fibrillation: the Fushimi Atrial Fibrillation Registry. Stroke 2015;46:3354-3361.

10 Hamatani Y, Ogawa H, Uozumi R, Iguchi M, Yamashita Y, Esato M, et al: Low body weight is associated with the incidence of stroke in atrial fibrillation patients - insight from the Fushimi AF Registry. Circ J 2015;79:10091017.

11 Yamashita Y, Hamatani Y, Esato M, Chun YH, Tsuji H, Wada H, et al: Clinical characteristics and outcomes in extreme elderly (age $\geq 85$ years) Japanese patients with atrial fibrillation: the Fushimi AF Registry. Chest 2016;149:401-412.

12 Kim SJ, Ryoo S, Kwon S, Park YK, Kim JP, Lee GY, et al: Is atrial fibrillation always a culprit of stroke in patients with atrial fibrillation plus stroke? Cerebrovasc Dis 2013;36:373-382.

13 Schiemanck SK, Post MW, Witkamp TD, Kappelle LJ, Prevo AJ: Relationship between ischemic lesion volume and functional status in the 2nd week after middle cerebral artery stroke. Neurorehabil Neural Repair 2005; 19:133-138

14 Group JJW: Guidelines for pharmacotherapy of atrial fibrillation (JCS 2013). Circ J 2014;78:1997-2021.

15 Ganesan AN, Chew DP, Hartshorne T, Selvanayagam JB, Aylward PE, Sanders P, et al: The impact of atrial fibrillation type on the risk of thromboembolism, mortality, and bleeding: a systematic review and meta-analysis. Eur Heart J 2016;37:1591-1602.

16 Capucci A, Santini M, Padeletti L, Gulizia M, Botto G, Boriani G, et al: Monitored atrial fibrillation duration predicts arterial embolic events in patients suffering from bradycardia and atrial fibrillation implanted with antitachycardia pacemakers. J Am Coll Cardiol 2005;46:1913-1920.

17 Boriani G, Botto GL, Padeletti L, Santini M, Capucci A, Gulizia M, et al: Improving stroke risk stratification using the CHADS2 and CHA2DS2-VASc risk scores in patients with paroxysmal atrial fibrillation by continuous arrhythmia burden monitoring. Stroke 2011;42:1768-1770.

18 Cambeiro G, Cristina M, Rodríguez-Mañero, Moisés, Raposeiras-Roubin, Assi A, et al: Review of obesity and atrial fibrillation: exploring the paradox. J Atr Fibrillation 2015;8:1259.

19 Connolly SJ, Ezekowitz MD, Yusuf S, Eikelboom J, Oldgren J, Parekh A, et al: Dabigatran versus warfarin in patients with atrial fibrillation. N Engl J Med 2009;361:1139-1151.

20 Patel MR, Mahaffey KW, Garg J, Pan G, Singer DE, Hacke W, et al: Rivaroxaban versus warfarin in nonvalvular atrial fibrillation. N Engl J Med 2011;365:883-891.

21 Granger CB, Alexander JH, McMurray JJ, Lopes RD, Hylek EM, Hanna M, et al: Apixaban versus warfarin in patients with atrial fibrillation. N Engl J Med 2011;365:981-992.

22 Novo G, Guttilla D, Fazio G, Cooper D, Novo S: The role of the renin-angiotensin system in atrial fibrillation and the therapeutic effects of ACE-Is and ARBS. Br J Clin Pharmacol 2008;66:345-351.

23 Suzuki S, Yamashita T, Okumura K, Atarashi H, Akao M, Ogawa H, et al: Incidence of ischemic stroke in Japanese patients with atrial fibrillation not receiving anticoagulation therapy: pooled analysis of the Shinken Database, J-RHYTHM registry, and Fushimi AF registry. Circ J 2015;79:432-438.

24 Warach S, Pettigrew LC, Dashe JF, Pullicino P, Lefkowitz DM, Sabounjian L, et al: Effect of citicoline on ischemic lesions as measured by diffusion-weighted magnetic resonance imaging. Citicoline 010 investigators. Ann Neurol 2000;48:713-722.

25 Huang M-J, Wei R, Wang Y, Su T, Di P, Li Q, et al: Blood coagulation system in patients with chronic kidney disease: a prospective observational study. BMJ Open 2017; 7:e014294.

26 Yahalom G, Schwartz R, Schwammenthal Y, Merzeliak O, Toashi M, Orion D, et al: Chronic kidney disease and clinical outcome in patients with acute stroke. Stroke 2009;40:1296-1303.

27 Di Lullo L, Ronco C, Cozzolino M, Russo D, Russo L, Di Iorio B, et al: Nonvitamin K-dependent oral anticoagulants (NOACs) in chronic kidney disease patients with atrial fibrillation. Thromb Res 2017;155:38-47.

28 Kleindorfer D, Kissela B, Schneider A, Woo D, Khoury J, Miller R, et al: Eligibility for recombinant tissue plasminogen activator in acute ischemic stroke: a population-based study. Stroke 2004;35:e27-e29.

29 Skolarus LE, Meurer WJ, Shanmugasundaram K, Adelman EE, Scott PA, Burke JF: Marked regional variation in acute stroke treatment among medicare beneficiaries. Stroke 2015;46:1890-1896.

30 Akao M, Chun YH, Esato M, Abe M, Tsuji H, Wada H, Hasegawa K, et al: Inappropriate use of oral anticoagulants for patients with atrial fibrillation: 1-year outcomes of the Fushimi AF Registry. Circ J 2014;78:2166-2172. 